\title{
Metodología de diseño, observación y cálculo de redes geodésicas exteriores para túneles de gran longitud
}

\section{Methedology for designing, observing and computing external geodetic networks of long lengths tunnels}

\author{
J. Velasco $^{(*)}$, T. Herrero ${ }^{(*)}$, J. Prieto $^{(*)}$
}

\section{RESUMEN}

La realización de túneles de gran longitud para ferrocarriles ha adquirido un gran auge en los últimos años. En España se han abordado proyectos de estas características, no existiendo para su ejecución una metodología completa y contrastada de actuación. Las características geométricas, de observación y de trabajo en túneles hace que las metodologías que se aplican en otros proyectos de ingeniería no sean aplicables por las siguientes causas: separación de las redes exteriores e interiores de los túneles debido a la diferente naturaleza de los observables, geometría en el interior siempre desfavorable a los requerimientos de observación clásica, mala visibilidad dentro del túnel, aumento de errores conforme avanza la perforación, y movimientos propios del túnel durante su ejecución por la propia geodinámica activa. Los patrones de observación geodésica usados deben revisarse cuando se ejecutan túneles de gran longitud. Este trabajo establece una metodología para el diseño de redes exteriores.

Palabras clave: Ferrocarriles; túneles; GNSS; geodesia; sistemas de referencia; redes geodésicas y topográficas.

\section{SUMMARY}

The realization of long railway tunnels has acquired a great interest in recent years. In Spain it is necessary to address projects of this nature, but ther is no corresponding methodological framework supporting them. The tunnel observational and working geometrical properties, make that former methodologies used may be unuseful in this case: the observation of the exterior and interior geodetical networks of the tunnel is different in nature. Conditions of visibility in the interior of the tunnels, regardless of the geometry, are not the most advantageous for observation due to the production system and the natural conditions of the tunnels. Errors increase as the drilling of the tunnel progresses, as it becomes problematical to perform continuous verifications along the itinerary itself. Moreover, inherent tunnel movements due to active geodynamics must also be considered. Therefore patterns for geodetic and topographic observations have to be reviewed when very long tunnels are constructed.

Keywords: Raiways; tunnels; GNSS; geodesy; reference systems; geodetic and land surveying networks.

(*) Universidad Politécnica de Madrid (España).

Persona de contacto/Corresponding author: jesus.velasco@upm.es (J. Velasco)

Cómo citar este artículo/Citation: Velasco, J., Herrero, T.,Prieto, J. (2014). Metodología de diseño, observación y cálculo de redes geodésicas exteriores para túneles de gran longitud. Informes de la Construcción, 66(533): e010, doi: http://dx.doi.org/10.3989/ic.13.007.

Licencia/License: Salvo indicación contraria, todos los contenidos de la edición electrónica de Informes de la Construcción se distribuyen bajo una licencia de uso y distribución Creative Commons Reconocimiento no Comercial 3.o. España (cc-by-nc). 


\section{INTRODUCCIÓN}

En toda obra de Ingeniería Civil existen fundamentalmente tres partes claramente diferenciadas: elaboración del anteproyecto, elaboración del proyecto definitivo y construcción. En la realización de estos tres grandes apartados, como son túneles de ferrocarril para trenes de alta velocidad, intervienen múltiples disciplinas. Una de ellas engloba los trabajos geodésicos y topográficos en los túneles que requieren establecer sistemas de referencias, metodología de observación, instrumental y cálculo (1).

En el caso de este estudio el objetivo es garantizar, desde un punto de vista geométrico, que la construcción de los túneles se encuentre dentro de las tolerancias admisibles para este tipo de obra. En este sentido se deben tener en cuenta determinadas cuestiones geodésicas, topográficas y cartográficas a la hora de desarrollar un proyecto de ingeniería tales como elección del sistema geodésico de referencia, elección del sistema de proyección cartográfica, implantación y observación de una red de precisión, metodología de observación e instrumentación a utilizar para finalmente establecer una estrategia de cálculo y compensación de las observaciones realizadas.

Para la realización de la obra se ha de materializar una red geodésica-topográfica que abarque toda la zona de actuación de tal forma que todos los trabajos a desarrollar posteriormente se referencian al Sistema Geodésico Oficial (2). Debido a que la toma de datos de campo para obtener la cartografía de la zona de trabajo se suele realizar mediante técnicas GNSS (Sistema Global de Navegación por Satélites) se necesita realizar una transformación entre un sistema geodésico de referencia global y un sistema geodésico de referencia local. Dicha transformación lleva consigo, por lo general, una pérdida de precisión en la determinación de los puntos de la red (3). Esta pérdida de precisión se eliminará cuando oficialmente se utilice el Sistema de Referencia Terrestre Europeo, ETRS89 (4), pero hasta que eso no ocurra, se seguirán arrastrando dichos errores que habrá que cuantificar y minimizar a la hora de la ejecución de la obra. La elección del sistema geodésico de referencia en el cual se va a trabajar es una cuestión importante. En la actualidad, los proyectos, según normativa del Ministerio de Fomento en España peninsular y en Baleares, están redactados en el sistema ED-50 (Datum Europeo 1950) (5), pero cuando se van a ejecutar los distintos tramos de la obra, a la hora de realizar el proyecto de la red exterior, se pueden presentar tres alternativas:

\section{Sistema geodésico de referencia local (Sistema de} Ingeniería): definido por un sistema de referencia específico para éste tipo de obras considerando un punto con coordenadas planimétricas arbitrarias, un acimut de referencia, una distancia y un origen de altitudes enlazado con la Red de Nivelación de Alta Precisión, o bien, dos puntos con coordenadas arbitrarias, obtenidas, por ejemplo, con técnicas de observación GNSS, con lo cual minimizamos los errores de la Red Geodésica y un origen de altitudes enlazado con la Red de Nivelación de Alta Precisión. Es decir, definir un DATUM local y propio para la obra (5).

2. Sistema geodésico de referencia global: materializado por un sistema de referencia global (ETRS89) a partir de observaciones mediante técnicas GNSS a los vértices de la red oficial REGENTE (Red GEodesica Nacional Téc- nicas Espaciales), cuya precisión nominal está por debajo de los 5 centímetros (6). Estas precisiones son mucho mejores que las de los vértices en el sistema ED50. La altimetría de los vértices de la red se obtendría a partir de la Red de Nivelación de Alta Precisión.

3. Sistema geodésico de referencia local oficial: El inconveniente de esta tercera solución es la perdida de precisión que se produce debido a los errores en la transformación motivados por la menor precisión de las coordenadas de los vértices geodésicos en el sistema ED50, que se necesitan para realizar dicha transformación, respecto a las coordenadas obtenidas a partir de las observaciones con técnicas GNSS.

Es importante indicar que a partir del primero de enero del 2015 toda la cartografía y bases de datos de información geográfica y cartográfica producida o actualizada por las Administraciones Públicas deberán realizarse en el sistema ETRS89. Hasta entonces se podrán utilizar los dos sistemas ED50 o ETRS89, siempre que las producciones en ED50 contengan la referencia a ETRS89 (5).

Según Braker (7) cuando se va a iniciar la fase de construcción hay que analizar las redes topográficas exteriores, lo cual comprende su diseño, metodología de observación, instrumentación a utilizar, así como el cálculo y compensación de dichas redes.

La cuestión más importante, desde el punto de vista geodésico y topográfico, en la construcción de un túnel es asegurar que el calado esté dentro de las tolerancias requeridas, normalmente un centímetro por kilómetro (8). Generalmente, en obras singulares como pueden ser túneles ferroviarios de gran longitud para trenes de alta velocidad, la excavación se suele realizar desde dos bocas a la vez, a fin de conseguir mayor velocidad en los trabajos de excavación, o bien, desde dos bocas a la vez y efectuando pozos ó rampas intermedios que permitan acceder desde la superficie a la rasante del proyecto. Para túneles de longitudes menores de $10 \mathrm{~km}$, la excavación se puede realizar desde una de las bocas.

La obtención de la componente altimétrica se determina de forma independiente mediante técnicas de nivelación de precisión, método que nos garantiza las precisiones requeridas. En cuanto a las redes planimétricas las podemos dividir en: red exterior (redes de superficie) y redes interiores (redes de túneles) debido a que tanto la geometría como la metodología de observación son distintas.

Definido los frentes desde los cuales se han de iniciar los túneles, hay que diseñar la red exterior de tal forma que englobe todas las bocas de los túneles garantizando fundamentalmente la homogeneidad de los acimutes de entrada a los mismos. En la actualidad dichas redes se observan mediante técnicas GNSS pues son más precisas, menos laboriosas y económicas que los métodos clásicos (9).

En éste trabajo se va a analizar las redes exteriores a los túneles y la metodología que a continuación se propone es fruto de un profundo análisis y estudio de los diversos factores que intervienen en la observación, tanto geodésica como topográfica, en un túnel así como la experiencia adquirida en los últimos ocho años en éste campo. 


\section{METODOLOGÍA}

Esta metodología comprende el diseño, observación de redes exteriores geodésicas-topográficas para la construcción de túneles de gran longitud para ferrocarriles de alta velocidad. Para ello es necesaria la utilización de una instrumentación adecuada. Estas cuestiones serán abordadas en los siguientes apartados y ha sido ensayada en la construcción de los Túneles TAV de Pajares. La zona comprende las comunidades de Castilla León y Principado de Asturias atravesando mediante el túnel diseñado y construido el Puerto de Pajares (Figura 1).

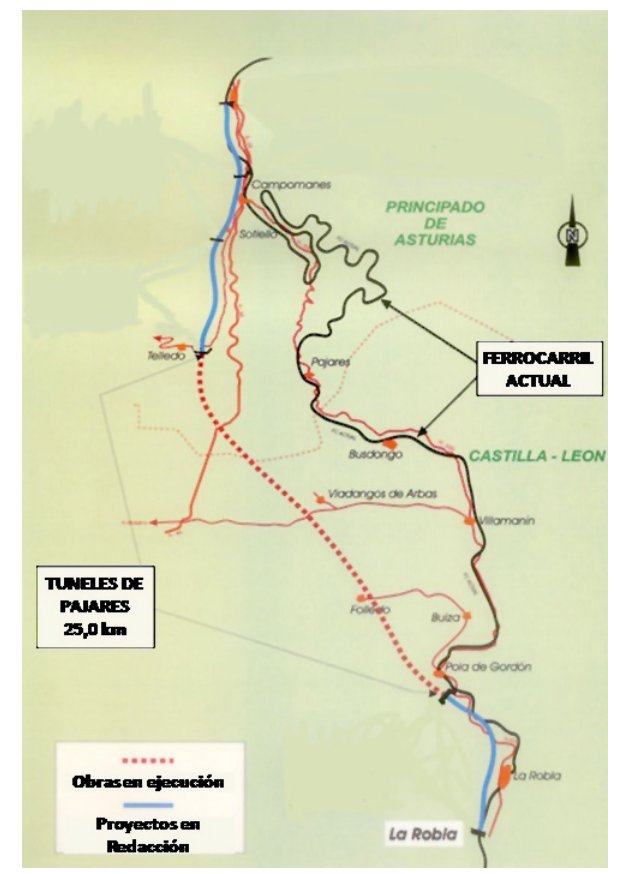

Figura 1.Trazados antiguo y nuevo.

\subsection{Diseño de la Red y Métodos}

Cuando se inicia una perforación simultánea desde dos o mas frentes, éstos han de encontrarse y coincidir en un determinado punto o perfil del túnel, por lo que es necesario que los puntos definitorios de las distintas bocas de excavación estén perfectamente enlazados, tanto planimétrica como altimétricamente. Esta primera fase se hace en la actualidad mediante técnicas GNSS.

Una de las ventajas de la utilización para la observación de la red exterior mediante técnicas GNSS es la no necesaria intervisibilidad entre los vértices que constituyen dicha red. Se minimizan así los errores que se transmitirían mediante una triangulación realizada por técnicas clásicas de observación. Además, el tiempo de la observación de la red usando GNSS se disminuye. En este sentido no hay que olvidar que dichas redes han de ser utilizadas para realizar observaciones con estaciones totales con el fin de transferir coordenadas al interior de los túneles y guiar la excavación, por lo que el diseño de las redes de las bocas ha de tener los mismos condicionantes geométricos que una red observada mediante técnicas clásicas:

- Intervisibilidad entre los vértices de cada red de bocas.

- Distancias homogéneas entre los vértices.

- Bondad de geometría de las redes.
Por lo general, las redes que se establecen para obtener la cartografía necesaria en la fase de redacción del proyecto del túnel suelen estar mal diseñadas para la posterior fase de ejecución: tienen un número insuficiente de vértices y carecen de la adecuada monumentación. Se deben tener en cuenta las siguientes cuestiones:

- Las redes deben abarcar todas las bocas que se van a utilizar para la perforación y materializar con vértices con monumentación adecuada, es decir, tipo pilar anclado al terreno siguiendo las normas de construcción de vértices del Instituto Geográfico Nacional, en terrenos estables desde el punto de vista geológico, con centrado forzado y ubicados en lugares de fácil acceso.

- La red exterior constituye el marco geodésico de referencia en el que se ha de apoyar la red interior la cual tendrá como fin principal el guiado de la tuneladoras. Por éste motivo su diseño, observación, cálculo y compensación adquiere una importancia fundamental.

- El diseño de la red se ha de realizar siguiendo las especificaciones geométricas de una red clásica, es decir, formando idealmente triángulos equiláteros en cada boca de entrada.

- Disponer al menos tres vértices por cada boca de entrada, en zonas geológicamente estables, uno de ellos situado en la prolongación teórica del eje del túnel visible desde los otros vértices.

En el caso de túneles en el que el calado se realice desde los dos extremos, la red exterior debería estar formada, al menos, por dos triángulos equiláteros pues los errores correspondientes son mínimos (10), es decir, tres vértices en cada boca. Primeramente se establece un vértice en la prolongación del eje teórico del túnel, denominado polo, y posteriormente, los otros dos vértices para cada boca formando un triángulo equilátero con el polo. Diseñar una red exterior con un número de vértices mayor no aporta, desde el punto de vista geodésico y topográfico, ninguna mejora de dicha red. Pero como en el transcurso de la ejecución de la obra desaparecerán visuales, es aconsejable ampliar las redes de cada boca de entrada con cuatro vértices según se muestra en la Figura 2. Tres vértices formando un triángulo equilátero y el cuarto situado en el baricentro de dicho triángulo y en la prolongación del eje teórico del túnel.

Realizado el diseño geométrico de la red, se tiene que analizar cual ha de ser la distancia entre los vértices de las redes de

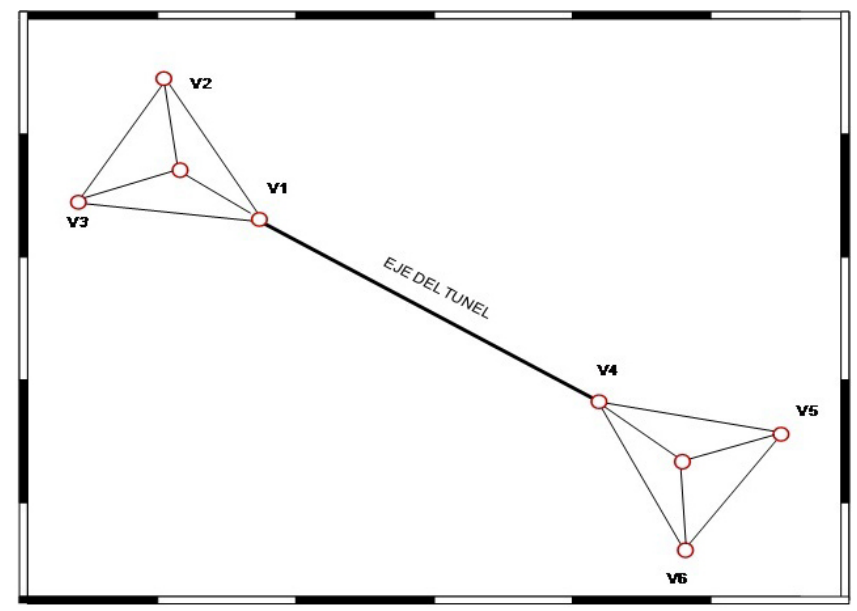

Figura 2. Diseño de Red Exterior. 
cada boca así como la distancia del polo de entrada a la boca del túnel. Las zonas donde se realizan este tipo de obras son montañosas, entonces, además de las limitaciones de visibilidad entre vértices se suele añadir condiciones meteorológicas adversas que afectarán a las observaciones que durante la ejecución de la obra se realizan con bastante frecuencia. Debido a este último factor, la distancia no debería ser mayor de 2500 metros. Si los vértices se situaran a la distancia de 2500 metros, la red admite ser observada perfectamente utilizando metodología RTK (Cinemático en Tiempo Real) por lo que el tiempo empleado en la observación de la red disminuiría considerablemente.

Si se supone que el vértice del polo de una de las bocas tiene error cero y que la precisión de una base línea observada con técnicas GNSS es de $3 \mathrm{~mm} \pm 1 \mathrm{ppm}$, para una distancia de $n$ kilómetros el error sería $0,1 \times n+0,3 \mathrm{~cm}$, es decir, para una distancia entre los vértices de los polos de las bocas de 30 kilómetros el error sería $35 \mathrm{~mm}$. Dicho error es equivalente para esa distancia, a un error angular de 1 segundo centesimal.

El error de orientación que se produce en cada entrada a los túneles es función del error del vértice situado en cada polo y del error de la red de dicha entrada. En el caso más desfavorable su valor es de 6 segundos centesimales, por lo que la transmisión de dicho error al primer vértice en el interior del túnel es de $10 \mathrm{~mm}$ en coordenadas.

La precisión obtenida mediante observaciones con técnicas GNSS, utilizando el método estático rápido relativo entre los polos de las dos bocas de túneles garantiza pues las precisiones requeridas en éste tipo de obras, pues está por debajo del centímetro.

Se puede afirmar que las técnicas de observación GNSS, método estático rápido, son las más adecuadas por tiempo, precisión y rendimiento respecto a otras técnicas de captura de datos y son las que se deben utilizar, previo establecimiento de una geometría idónea de los vértices de las redes de las bocas de entrada a los túneles.

Demostrada la idoneidad de los sistemas GNSS para éste tipo de redes, se plantea analizar a continuación las siguientes cuestiones:

- Número de receptores a utilizar en la observación de la red.

- Tiempo de observación en cada sesión de observación.

- Diseño de observación.

En cuanto al número de receptores a utilizar, en principio es aleatorio. Variará desde un mínimo de dos hasta un máximo igual al número de puntos de la red exterior más los vértices geodésicos que se consideren necesarios para una posterior transformación al sistema ED50, si fuese necesario. Si bien en principio el número de receptores es variable, dicho número está condicionado entre otros factores por la disponibilidad de aparatos y operadores que los tienen que manejar. En cuanto al tiempo idóneo de observación en cada vértice, los cálculos que se han realizado en múltiples redes de estas características como se verá posteriormente, se puede fijar en una hora. Establecido el número de receptores que van a intervenir en la campaña y el tiempo de observación de los vértices de la red exterior, se debe establecer un protocolo y un diseño del protocolo de observación de dicha red.
Existen algoritmos topológicos de diseño de observación de redes utilizando técnicas GNSS, como los desarrollados por Snay (11) ó Unguendoli (12), los cuales se ensayan a continuación. Para ello se parte de las algunas hipótesis:

La red que va a ser diseñada para su observación será homogénea en el sentido de que todos los vértices serán observados el mismo número de veces $(n)$.

El número de sesiones de observación viene dado por la ecuación [1].

$$
S=n \times m / 2
$$

siendo $n$ el número de vértices y $m$ el número de observaciones por vértice.

El número de ecuaciones independientes, o líneas base, viene dado por la ecuación [2].

$$
b=(r-1) \times s=(n \times m / r)
$$

siendo en este caso $r$ el número de receptores disponibles.

Una vez diseñada la red y después de seleccionar el número de estacionamientos por vértice, se debe considerar la geometría de la red.

De los distintos algoritmos existentes se ha elegido el de Lazo (12) que tiene la peculiaridad de eludir la observación de una línea base más de una vez. Dos vértices no pueden estar contenidos en más de una sesión común. Esto significa que la red contiene líneas bases distintas en número de [3].

$$
(r-1) \times n \times m / 2
$$

Además, favorece una elección para líneas base cortas que van a ser observadas lo cual reduce el tiempo de desplazamiento entre las sucesivas sesiones y además se homogeniza la distancia media entre vértices, aunque algunas observaciones de líneas base más largas fortalecen la red controlando la acumulación del error con la distancia.

Por otra parte, el IGN diseñó una metodología de observación para la red REGENTE, observando en método estático simultáneamente bloques de nueve vértices con nueve receptores bifrecuencia constituyendo, sucesivamente, polígonos adosados con tres estaciones comunes de manera que cada estación se observa al menos en dos bloques diferentes. Cada bloque se observa en dos sesiones diferentes de 3 horas de duración, imponiendo, por lo general que una sesión sea matutina y otra vespertina para obtener en cada una geometrías de constelación totalmente diferentes (13).

Otro apartado dentro del diseño de la observación es analizar la máscara de elevación que se debe introducir en los receptores para evitar efectos multipath y pérdidas de calidad de señal. En proyectos realizados en la ETSI Topografía, Geodesia y Cartografía con diferentes máscaras de elevación, se ha llegado a la conclusión que es preferible usar máscara de 10 grados de elevación a fin de obtener la precisión requerida (14)

En cuanto al tiempo de registro de las observaciones GNSS, intervalo entre épocas, con análisis realizados en otros estu- 
dios (15) se llega a la conclusión que utilizar intervalos de 5 segundos, 10 segundos y 15 segundos no cambia el resultado del cálculo de la línea base.

Como puede apreciarse, existen múltiples maneras de diseñar la observación con técnicas GNSS que la optimizan, pero desde nuestro punto de vista una condición fundamental y que siempre se debe cumplir es que todos los vértices han de ser observados al menos dos veces, garantizando así la repetibilidad y fiabilidad.

Observar tres veces cada vértice no mejora cualitativamente las coordenadas finales y aumenta el tiempo de observación de la red. Hay que mencionar que la red exterior está por lo general sobrediseñada, y los requerimientos en cuanto a su precisión sobreestimada, mientras que en las redes interiores la precisión es infravalorada. El esfuerzo debe estar dirigido a la optimización y mejora del diseño de la red en el interior de los túneles (16). Es decir, siendo importante el diseño y la observación de la red exterior, debido a las características singulares de éste tipo de obras, es más importante, como se tratará en trabajos posteriores, el diseño y observación de las redes interiores, pues los errores en estas redes siempre son de mayor magnitud. Esta cuestión no será tratada en este estudio.

\subsection{Aplicación a los Túneles TAV de Pajares}

Para la realización de Túneles de Pajares, debido a que la perforación se realizó desde tres frentes se diseñó una red exterior constituida, en principio, por 12 vértices. Cuatro por cada boca de entrada, según los criterios establecidos en el apartado anterior, perfectamente monumentados según las normas de construcción de vértices del $(I G N)$, incluyendo un centrado forzado (Figura 3).

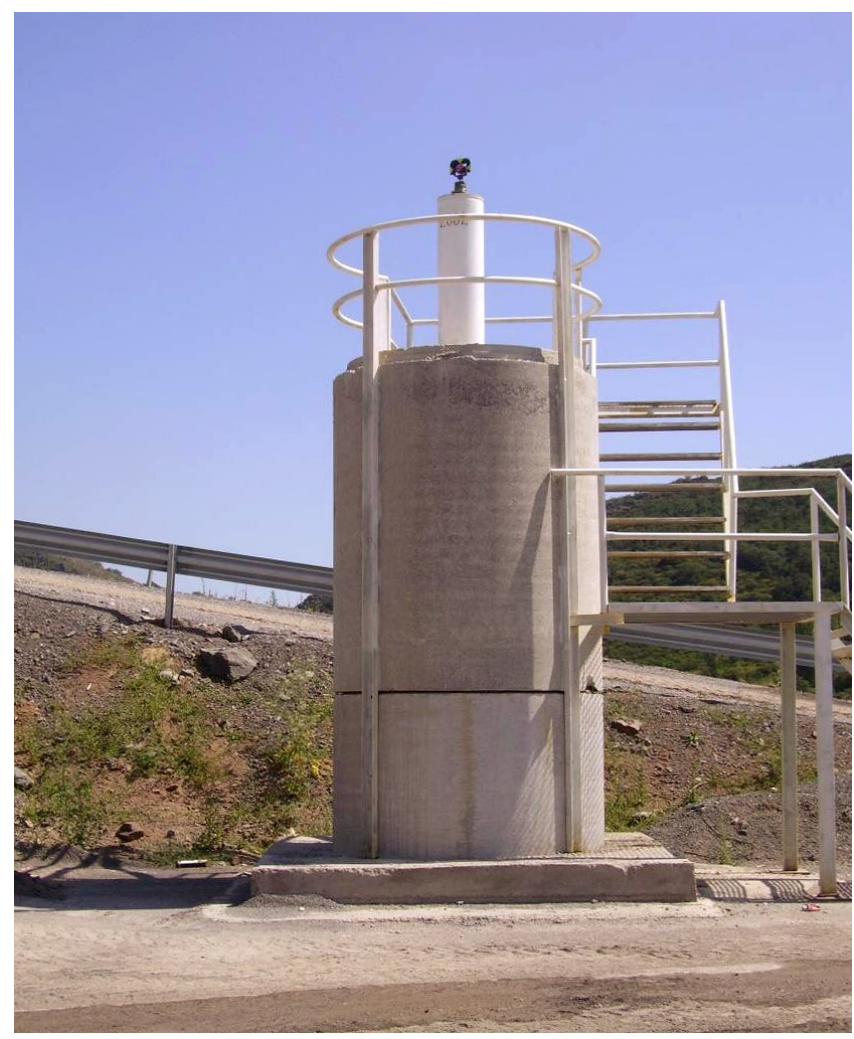

Figura 3. Vértice Red de Pajares (Boca Norte).
Para la observación de dicha red mediante técnicas GNSS, se planificó además el enlace con ocho vértices de la red geodésica española, cinco de los cuales pertenecen a la red REGENTE. El motivo de la elección de dichos vértices geodésicos fue el haber sido utilizados en la ejecución de la cartografía de proyecto. De esta forma, la transformación entre el sistema ETRS89 y el sistema geodésico local de referencia, ED50, se haría a partir de dichos vértices, ajustándose de esta manera lo mejor posible a la cartografía existente de proyecto.

Para la observación de las redes exteriores se han de utilizar receptores bifrecuencia con medidas de código y fase sobre las portadoras L1 y L2. El método de observación elegido fue el estático relativo con cálculo en postproceso. La duración de cada sesión quedó establecida en 3 horas, con el fin de poder realizar posteriormente estudios comparativos acerca del tiempo idóneo de observación para redes de estas características. En relación al instrumental utilizado y tras un estudio previo se estimó la conveniencia de utilizar para la observación de la red 6 receptores bifrecuencia, los cuales en este caso fueron de la marca ASHTECH modelo Z-FX. En principio la observación fue diseñada siguiendo el algoritmo de Lazo propuesto de Unguendoli (12) en función del número de vértices $(n=20)$, número de receptores $(\mathrm{r}=6)$, número de observaciones por vértices $(\mathrm{m}=2)$.

Debido a la dificultad de acceso a una serie de vértices (más de dos horas de subida en alguno de ellos) se tuvo que rediseñar la observación, evitando subir a los vértices de mayor dificultad más de una vez, aunque con la premisa de observar dichos vértices en dos sesiones distintas.

Por último, añadir que la observación se realizó finalmente siguiendo el siguiente diseño:

a. Observación simultánea desde cuatro vértices geodésicos a dos vértices de cada red exterior de las bocas de entrada.

b. Observación simultánea desde dos vértices geodésicos a los cuatro vértices de cada red exterior de las bocas de entrada.

c. Observación simultánea de seis vértices, dos de cada boca de entrada.

d. Observación simultánea de los otros seis vértices, dos de cada boca de entrada.

e. Observación simultánea desde los cinco vértices REGENTE a cada uno de los vértices situados en los polos de las diferentes bocas de entrada.

La máscara de elevación fue de $10^{\circ} \mathrm{y}$ el intervalo de tiempo de registro de 10 segundos. De los diferentes métodos de observación existentes en las actualidad, técnicas GNSS y técnica clásicas de observación, y tras analizar las ventajas e inconvenientes de dichos métodos, se llega a la conclusión, que en la actualidad la metodología a utilizar ha de ser GNSS.

Esto nos lleva a realizar una clasificación entre las observaciones exteriores al túnel y las observaciones en el interior del túnel, pues obviamente y en la actualidad, en el interior no se pueden utilizar técnicas GNSS.

Todos los aspectos metodológicos tratados en esta sección se resumen en el diagrama de la Figura 4. 


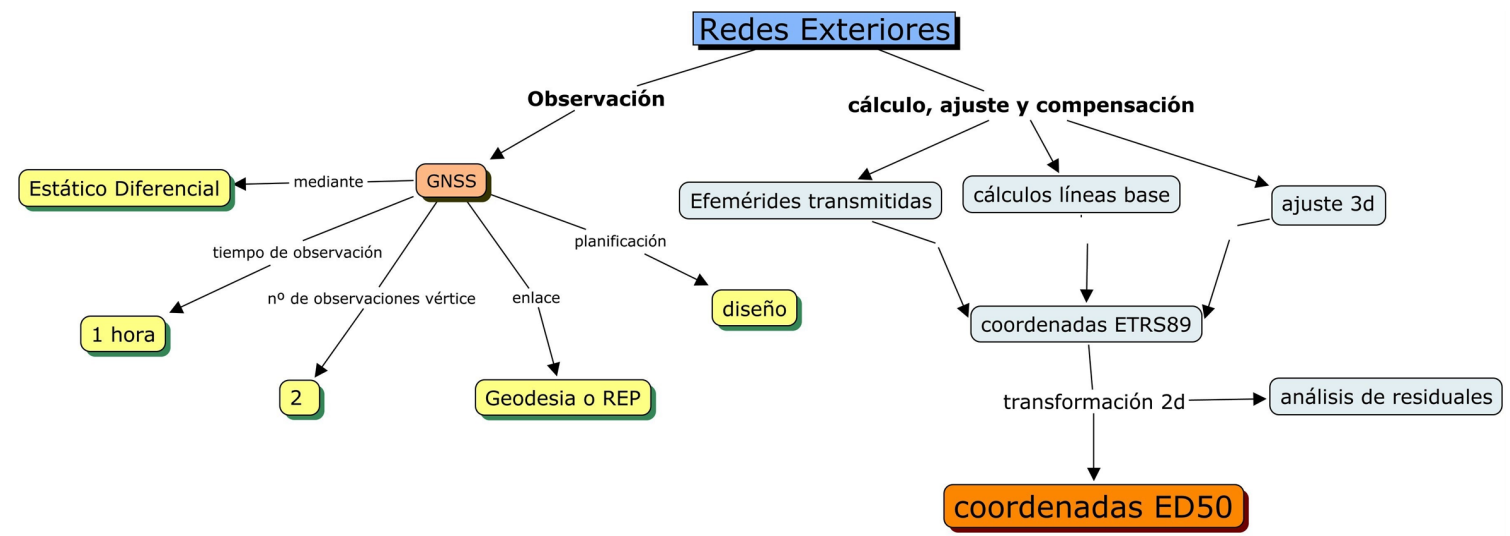

Figura 4. Diagrama de observación y cálculo de redes exteriores.

\section{RESULTADOS Y DISCUSIÓN}

En esta sección se presentan los resultados obtenidos para el establecimiento de la red geodésica exterior, su observación y cálculo según se ha explicado en la metodología expuesta anteriormente. Igualmente, se discuten los aspectos más relevantes que se deducen de las operaciones realizadas en cada una de las fases contempladas.

\subsection{Red diseñada y observada}

En primer lugar y en función de la precisión nominal del instrumental a utilizar (Estación Total) en la observación de las redes interiores, se ha de calcular la distancia mínima idónea entre los vértices. Partiendo de las siguientes hipótesis:

a. La precisión angular nominal de la estación total que se va a utilizar en la observación de las redes interiores es de 3 segundos centesimales.

b. La observación de las redes exteriores se realiza con técnicas GNSS.

La distancia entre vértices viene determinada por la primera condición, es decir, por la precisión del aparato a utilizar.

A partir del cálculo de la transmisión de errores en el ámbito de la teoría clásica que los describe, Bjethammar (17), la distancia mínima idónea entre vértices es de 1000 metros, pues las técnicas de observación GNSS, nos garantizan a éstas distancias las precisiones requeridas. En la Figura 5 se muestran los resultados obtenidos a partir de los valores de la Tabla 1 y se le han añadido los errores en distancia utilizando el método estático relativo GNSS.

Esto demuestra que si se utiliza el método estático relativo con postproceso para la observación de las redes de bocas con vértices situados a una distancia de 750 metros, se garantiza que la precisión en la orientación de la estación total necesaria para la observación de las redes interiores está dentro de la precisión de dicho instrumento.

Lógicamente, el vértice situado en el polo de entrada también debe estar situado a esa distancia respecto de la boca del túnel. En cuanto a la distancia máxima, viene condicionada fundamentalmente por la propia operatividad de la red y de la orografía de la zona donde se ejecuta la obra.

En este trabajo el diseño de observación en función del número de vértices, receptores y observaciones en cada vértice, se establece inicialmente de la siguiente manera: número de vértices $\mathrm{n}=20$, número de receptores $\mathrm{r}=6$, número de observaciones por vértices $m=2$.

De esta forma, el número de sesiones de observación resultantes son $s=(20 \times 2) / 6 \approx 7$. Debido a lo comentado en la metodología aplicada, el número de sesiones aumentó de 7 a 11, pero la campaña de observación se hizo en menos tiempo que el que se hubiera empleado si se hubiera realizado siguiendo el diseño teórico de la observación. Las sesiones de observación establecidas se muestran en la Tabla 1.

\section{Comparación errores EETT-GNSS}

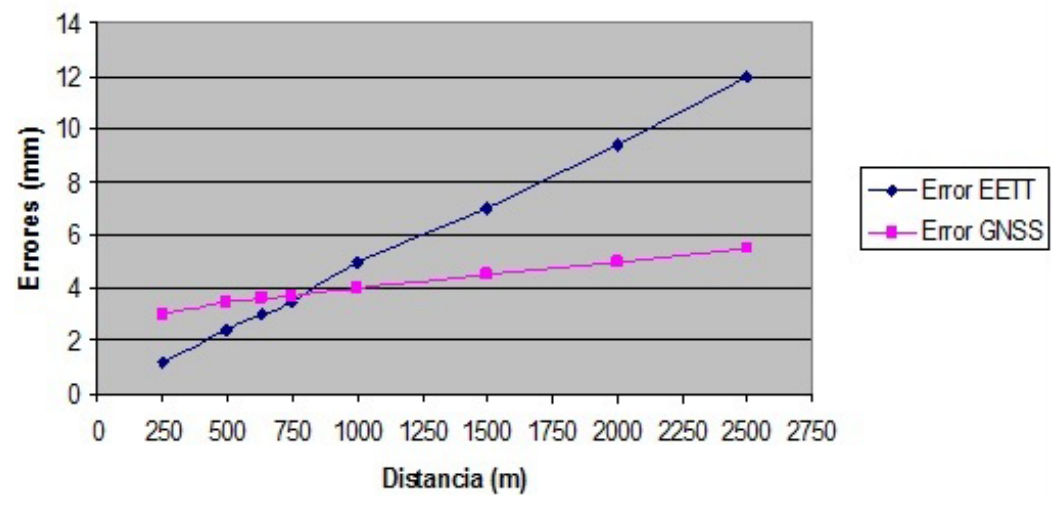

Figura 5. Precisión en función de la distancia. 
Tabla 1.

Diseño de observación. Red exterior tuneles de Pajares.

\begin{tabular}{|c|c|c|c|c|c|c|}
\hline RECEPTORES & R1 & R2 & R3 & R4 & R5 & R6 \\
\hline SESION1 & 901 & 902 & 951 & 952 & 103 & 104 \\
\hline SESION2 & 951 & 952 & 101 & 102 & 103 & 104 \\
\hline SESION3 & 903 & 951 & 952 & 953 & 201 & 203 \\
\hline SESION4 & 951 & 953 & 201 & 202 & 203 & 204 \\
\hline SESION5 & 903 & 904 & 905 & 953 & 302 & 303 \\
\hline SESION6 & 904 & 953 & 301 & 302 & 303 & 305 \\
\hline SESION7 & 102 & 104 & 202 & 204 & 302 & 305 \\
\hline SESION8 & 101 & 103 & 201 & 203 & 301 & 303 \\
\hline SESION9 & 901 & 902 & 903 & 904 & 905 & 102 \\
\hline SESION10 & 901 & 902 & 903 & 904 & 905 & 203 \\
\hline SESION11 & 901 & 902 & 903 & 904 & 905 & 305 \\
\hline
\end{tabular}

Esta red se diseñó de la siguiente forma. En la Boca Norte de perforación del túnel se han materializado los vértices 101, 102, 103 y 104. En la Zona Intermedia de perforación se encuentran los vértices 201, 202, 203 y 204 y en la Boca Sur del túnel se localizan los vértices 301, 202, 303 y 305. En la Figura 6 se muestra dicha red exterior así como su enlace con la Red Geodésica Nacional.

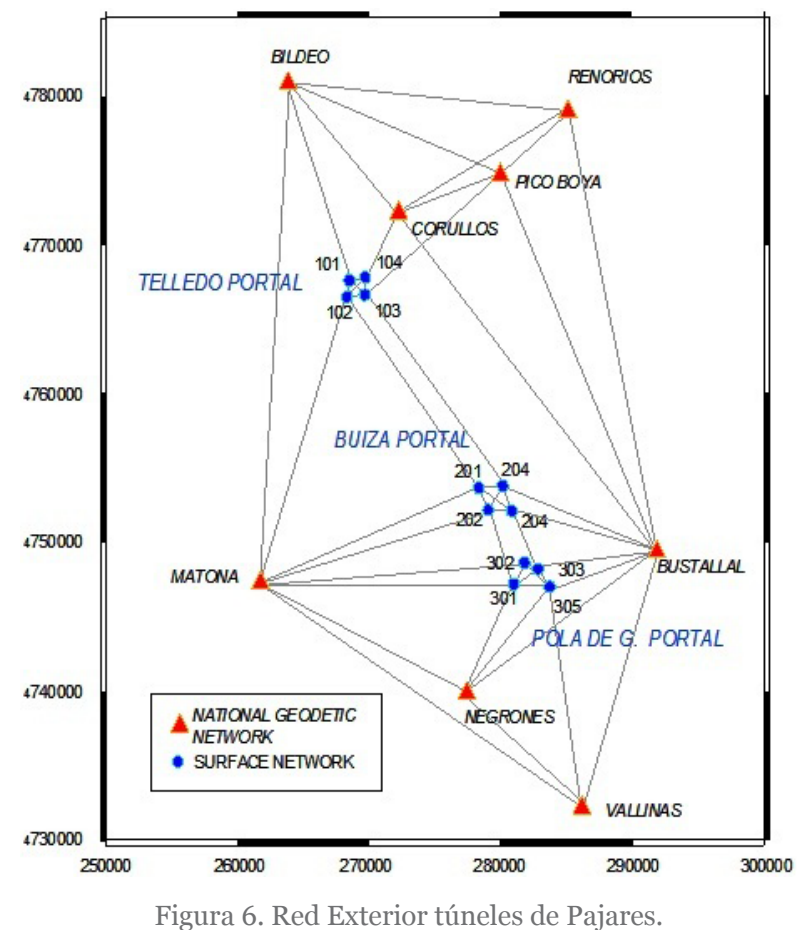

\subsection{Cálculos}

Los cálculos de las líneas bases se realizaron con el programa SKI-PRO de Leica-Geosystems y para la compensación de las líneas bases, se utilizó además del programa anterior el programa técnico-científico GEOLAB 2001.

A partir de los datos obtenidos de las observaciones se llevaron a cabo diferentes cálculos de la Red Exterior aplicando los siguientes criterios:

a. Cálculo de todas las líneas base con efemérides precisas.

b. Cálculo de todas las líneas base con efemérides transmitidas. c. Cálculo únicamente con líneas base independientes:

d. Cálculo con 2 horas de observación.

e. Cálculo con 1 hora de observación.

f. Cálculo con 30 minutos de observación.

g. Cálculo desde estaciones permanentes de referencia

A continuación se presentan bajo formas de tablas algunos de los resultados obtenidos a partir de los distintos cálculos realizados de la red exterior. En la Tabla 2 se muestran las diferencias obtenidas entre el cálculo realizado utilizando efemérides precisas y efemérides transmitidas. Como se muestra en dicha tabla utilizar unas efemérides u otras no cambia significativamente la precisión de las coordenadas.

En los últimos años, cada vez está más extendido el uso de Redes Activas de Estaciones Permanentes GNSS (18). Debido a ello también he realizado un cálculo a partir de los datos de observación de dichas Estaciones Permanentes. Las diferencias encontradas entre el cálculo con todas las líneas bases y el cálculo desde dichas Estaciones se puede ver en la Tabla 3 .

Como se puede ver en la Tabla 3, las diferencias de coordenadas en $(\mathrm{X}, \mathrm{Y})$ no empeoran utilizando estaciones permanentes de referencia. Así, la utilización de estaciones permanentes de referencia supondrá un considerable ahorro de tiempo y de instrumental, fundamentalmente cuando entre en vigor el sistema ETRS89.

\subsection{Discusión de los Resultados}

Como consecuencia de estos resultados obtenidos, se plantean por tanto, las siguientes cuestiones:

- ¿Cuál es la metodología más apropiada para la observación?

- ¿Qué características técnicas deben tener la instrumentación a utilizar?

- ¿Cuál debe ser el diseño geométrico de red, tanto exterior como interior, para minimizar los errores de las observaciones?

- ¿Cuál debe ser la distancia óptima entre vértices de las redes exteriores?

- ¿Qué criterios de cálculo, ajuste y compensación que deben aplicarse a las observaciones realizadas?

- ¿Cómo se debe realizar la transformación entre los distintos sistemas de referencia? 
Tabla 2.

Diferencias de coordenadas, en metros, utilizando efemérides transmitidas y precisas.

\begin{tabular}{|c|c|c|c|c|c|}
\hline VERTICES & $\boldsymbol{\Delta X}$ & $\boldsymbol{\Delta Y}$ & $\boldsymbol{\Delta h}$ & $\boldsymbol{\sigma}(\mathbf{X}, \mathbf{Y})$ & $\boldsymbol{\sigma}(\mathbf{h})$ \\
\hline VALLINAS & 0,000 & 0,000 & $-0,001$ & 0,005 & 0,007 \\
\hline RENORIOS & $-0,001$ & 0,001 & $-0,001$ & 0,005 & 0,007 \\
\hline PICO BOYA & 0,000 & 0,000 & 0,000 & 0,005 & 0,007 \\
\hline NEGRONES & 0,001 & 0,001 & 0,000 & 0,004 & 0,007 \\
\hline MATONA & 0,000 & 0,000 & 0,000 & 0,000 & 0,000 \\
\hline CORULLOS & 0,000 & 0,000 & 0,000 & 0,005 & 0,007 \\
\hline BUSTALLAL & 0,001 & 0,000 & 0,000 & 0,004 & 0,007 \\
\hline BILDEO & 0,001 & 0,001 & 0,001 & 0,005 & 0,007 \\
\hline $\mathbf{3 0 5}$ & 0,002 & 0,000 & 0,000 & 0,005 & 0,007 \\
\hline $\mathbf{3 0 3}$ & 0,002 & 0,000 & 0,001 & 0,004 & 0,007 \\
\hline $\mathbf{3 0 2}$ & 0,002 & 0,000 & 0,000 & 0,004 & 0,007 \\
\hline $\mathbf{3 0 1}$ & 0,002 & 0,000 & 0,001 & 0,005 & 0,008 \\
\hline $\mathbf{2 0 4}$ & 0,001 & 0,000 & 0,000 & 0,005 & 0,007 \\
\hline $\mathbf{2 0 3}$ & 0,001 & 0,000 & 0,000 & 0,004 & 0,007 \\
\hline $\mathbf{2 0 2}$ & 0,001 & 0,000 & 0,000 & 0,005 & 0,007 \\
\hline $\mathbf{2 0 1}$ & 0,001 & 0,000 & 0,000 & 0,004 & 0,007 \\
\hline $\mathbf{1 0 4}$ & 0,001 & 0,000 & 0,000 & 0,005 & 0,008 \\
\hline $\mathbf{1 0 3}$ & 0,001 & 0,000 & 0,000 & 0,005 & 0,008 \\
\hline $\mathbf{1 0 2}$ & 0,001 & 0,000 & 0,000 & 0,005 & 0,008 \\
\hline $\mathbf{1 0 1}$ & 0,001 & 0,000 & 0,000 & 0,006 & 0,009 \\
\hline & & & & & \\
\hline
\end{tabular}

Tabla 3.

Diferencias de coordenadas, en metros, entre el cálculo utilizando 3 horas de observación y el cálculo realizado a partir de Estaciones Permanentes.

\begin{tabular}{|c|c|c|c|c|c|}
\hline VERTICE & $\boldsymbol{\Delta X}$ & $\boldsymbol{\Delta Y}$ & $\boldsymbol{\Delta h}$ & $\boldsymbol{\sigma}(\mathbf{X}, \mathbf{Y})$ & $\boldsymbol{\sigma}(\mathbf{h})$ \\
\hline $\mathbf{3 0 5}$ & $-0,007$ & 0,007 & $-0,027$ & 0,005 & 0,008 \\
\hline $\mathbf{3 0 3}$ & $-0,006$ & 0,008 & $-0,025$ & 0,005 & 0,008 \\
\hline $\mathbf{3 0 2}$ & $-0,007$ & 0,008 & $-0,026$ & 0,005 & 0,008 \\
\hline $\mathbf{3 0 1}$ & $-0,006$ & 0,007 & $-0,025$ & 0,005 & 0,008 \\
\hline $\mathbf{2 0 4}$ & $-0,005$ & 0,007 & $-0,025$ & 0,005 & 0,008 \\
\hline $\mathbf{2 0 3}$ & $-0,004$ & 0,007 & $-0,023$ & 0,005 & 0,007 \\
\hline $\mathbf{2 0 2}$ & $-0,005$ & 0,007 & $-0,024$ & 0,005 & 0,008 \\
\hline $\mathbf{2 0 1}$ & $-0,005$ & 0,007 & $-0,023$ & 0,005 & 0,007 \\
\hline $\mathbf{1 0 4}$ & $-0,003$ & 0,002 & $-0,034$ & 0,005 & 0,008 \\
\hline $\mathbf{1 0 3}$ & $-0,005$ & 0,003 & $-0,037$ & 0,006 & 0,009 \\
\hline $\mathbf{1 0 2}$ & $-0,001$ & 0,004 & $-0,029$ & 0,006 & 0,009 \\
\hline $\mathbf{1 0 1}$ & 0,001 & 0,002 & $-0,029$ & 0,006 & 0,010 \\
\hline
\end{tabular}

- ¿De qué manera afectan los errores de la transformación a las coordenadas finales de las redes?

A partir del análisis realizado, se observa que no existe una metodología establecida que optimice la forma de diseñar una observación para este tipo de redes, la cual puede ser extrapolable a otro tipo de redes de análogas características, pues, tanto el número de receptores como el tiempo de observación y las longitudes de los vértices son valores indeterminados. De esta forma, sobre el diseño geométrico, diseño de observación, tiempo de observación y cálculo de redes exteriores utilizando técnicas GNSS, habrá que tener en cuenta las siguientes pautas de trabajo: a. Desde el punto de vista geométrico la configuración idónea de las redes exteriores cuyo fin es servir de marco de referencia a las redes interiores es, en principio, igual que en el caso de redes observadas mediante técnicas clásicas, la de un triángulo equilátero. El problema que surge a la hora de materializar una red que cumpla las características anteriormente expuesta es el de la orografía del terreno donde se materializa dicha red. Cuando por motivos orográficos, no se puedan cumplir las condiciones geométricas ideales, a la hora de trasmitir la orientación desde los vértices situados en los polos de las diferentes bocas a las redes interiores se deben utilizar aquellas de mayor longitud utilizando los 
de menor distancia a los polos de las diferentes bocas para comprobar dicha orientación o bien se debe introducir un peso en dicha medida con el fin de homogeneizar las observaciones. Dichas redes deben quedar perfectamente monumentadas, con centrado forzado, en zonas de terreno estable y, siempre que sea posible, de fácil acceso. Muchos de los problemas de encaje de obras vienen motivados por los distintos vértices geodésicos que se utilizan, por lo que en España, para la construcción de líneas de ferrocarril para trenes de Alta Velocidad, el ADIF (Administrador de Infraestructuras Ferroviarias) en su pliego de condiciones técnicas exige la construcción perfectamente monumentada con hitos de construcción similar a los vértices geodésicos, observados con técnicas GNSS y nivelados mediante nivelación geométrica de precisión, de tal forma que dicha red constituye el Marco de Referencia para toda la línea de ferrocarril.

b. En el diseño de la observación habrá que incluir los vértices geodésicos necesarios que nos permiten encuadrar en el Marco de Referencia elegido nuestras observaciones y siempre que esto sea posible, los vértices existentes del proyecto de ejecución de la obra. Los vértices geodésicos serán, siempre que su geometría respecto a las redes exteriores sea la idónea, los utilizados cuando se realizó la cartografía de la fase de proyecto, la cual está referenciada a esos vértices. Como ya hemos comentado hasta el año 2015 se podrán utilizar como Sistemas de Referencia ED50 y ETRS89, a partir de dicho año toda la cartografía deberá estar referenciada al último sistema mencionado. $\mathrm{Si}$ el Sistema de Referencia elegido es ETRS89, la observación de las redes exteriores se puede realizar enlazándola con la Red de Estaciones Permanentes del Instituto Geográfico Nacional o con otras Redes de Estaciones Permanentes oficiales de las distintas Comunidades o de otros organismos que tengan el refrendo de sus coordenadas por el IGN. Esto hace que podamos minimizar el número de receptores a utilizar y evita el tener que visitar vértices geodésicos por lo que se optimiza la observación.

c. Los cálculos realizados con efemérides precisas y transmitidas no aportan mayor precisión a la hora del cálculo, por lo que es indiferente utilizar unas efemérides y otras. Si bien, debido a que el cálculo, se realiza en postproceso, no supone ningún esfuerzo utilizar las efemérides precisas para calcular.

d. Los cálculos realizados según se muestran en las tablas no ofrecen diferencia, por lo que es indistinto calcular todas las líneas bases que se forman ó únicamente las líneas bases linealmente independientes.

e. Respecto a los tiempos de observación, y tras analizar en las diferentes redes los cálculos obtenidos con tiempos que van desde tres horas a cálculos con una hora como figuran en las tablas anteriores. Concluir que el tiempo óptimo para realizar observaciones de redes de éstas características es de una hora. Observaciones de más tiempo no influye cualitativamente en la precisión de las coordenadas.

f. Un factor que hay que tener en cuenta es la fiabilidad de nuestras observaciones o capacidad que se tiene en el cálculo para detectar errores groseros, por lo que cada vértice de la red debe ser observado dos veces.

g. Si bien es necesario tener una red que garantice fundamentalmente la homogeneidad de los acimutes de entrada por las diferentes bocas desde las que se acomete este tipo de obras, lo cual se consigue con un diseño óptimo de la red y diseño de observación con las técnicas GNSS, el mayor esfuerzo, debe realizarse en la calidad de la observación de las redes interiores:
A modo de ejemplo y en base a lo anteriormente expuesto se pueden considerar las siguientes afirmaciones:

- Si suponemos al 95\% de confianza que el error de la red exterior es del orden de $50 \mathrm{~mm}($ ee $=50 \mathrm{~mm})$ y el error en la red interior es de $125 \mathrm{~mm}$ ( $\mathrm{ei}=125 \mathrm{~mm}$ ), la componente total del error vendría dada por: $\mathrm{e}=135 \mathrm{~mm}$.

- Reduciendo el error de la red exterior a $30 \mathrm{~mm}(\mathrm{ee}=30 \mathrm{~mm})$, lo que supone un aumento de la precisión del rango del $40 \%$, de dicha red, el error final sería de: e $=129 \mathrm{~mm}$. Esto supone sólo una mejora del $4 \%$.

- Sin embargo si reducimos el error de la red interior a $100 \mathrm{~mm}(\mathrm{ei}=100 \mathrm{~mm})$, lo que supone un aumento en la precisión del 20\%, el error final es: e = $112 \mathrm{~mm}$. Esto supone una mejora del 17\% tal como indica Fowler (8).

- Como ya se comentado la red exterior está normalmente sobrediseñada y las precisiones exigidas muy elevadas.

- La optimización para la precisión del error de calado del túnel debe concentrarse en la optimización y mejora del diseño de la red interior (16).

\subsection{Recomendaciones para el diseño, observación y cálculo de redes exteriores}

Estas recomendaciones se podrían resumir en:

1. Diseñar redes de al menos tres vértices en cada boca de los túneles con lados cuya distancia no sea menor de 750 metros ni mayor de 2,5 kilómetros.

2. Un vértice de cada boca ha de estar situado en la prolongación teórica del eje de los túneles a una distancia no menor de 1kilómetro de la entrada a dichos túneles.

3. Los otros dos vértices que forman la red de cada boca deben formar un triángulo, siendo idealmente mejor equilátero. Por la experiencia acumulada en las redes exteriores realizadas en túneles puede ser conveniente ampliar la red de cada emboquille del túnel con un cuarto vértice.

4. Observaciones con un número de receptores (seis es un número óptimo) que sean adecuados en función de la operatividad de recursos tanto humanos como de infraestructuras necesarias.

5. Monumentación en zonas geológicamente estables y siguiendo las normas establecidas por el IGN para la construcción de vértices geodésicos, y con centrado forzado.

6. Todos los vértices deben ser observados dos veces con el método de observación estático (ó estático rápido), el tiempo de observación será de 1 hora al menos, la máscara de elevación para los satélites de $10^{\circ}$ y el intervalo de observación de 10 segundos.

7. El cálculo de las líneas base es indistinto hacerlo con efemérides precisas o efemérides transmitidas.

\section{CONCLUSIONES}

Se presenta aquí una metodología desarrollada en la cual se analizan las distintas fases que, desde el punto de vista de los autores, constituyen los aspectos fundamentales para la elaboración de una metodología de diseño, observación, cálculo y compensación de redes exteriores para la construcción de túneles de gran longitud para ferrocarriles de alta velocidad. Se fundamenta en un profundo estudio de los diferentes temas planteados por los autores unido a una serie de cálculos en modo de simulaciones matemáticas y de datos obtenidos en campo, que han permitido extrapolar las experiencias en algunos túneles a caso generales. 
- No existe una metodología estructurada y mucho menos contrastada que comprenda todos los campos geodésicos y topográficos para la realización de túneles de gran longitud para ferrocarriles de alta velocidad.

- La elección del sistema geodésico de referencia es una cuestión importante a la hora de la realización de la obra

- Las características tan singulares de los túneles hace necesario diferenciar, desde el punto de vista de la topografía y geodesia, entre el exterior y el interior de los mismos. Tanto la geometría como los métodos de observación son diferentes dentro y fuera de los túneles, por lo que hay que separar las redes en el exterior de las redes en el interior. Lógicamente han de estar perfectamente enlazadas entre ellas puesto que forman una única red en el conjunto del proyecto.

- Cuando por motivos orográficos los vértices de la red exterior no cumplan las premisas de distancias entre ellos, es decir, las distancias no sean homogéneas, se ha de utilizar como vértice de orientación aquel que esté situado a mayor distancia del polo de entrada. Las orientaciones a los vértices que estén a menor distancia se utilizarán con su ponderación correspondiente para no perjudicar la calidad de la orientación.

\section{REFERENCIAS}

(1) Priego, E. (2009). Túneles y tuneladoras. Valencia: Ed. Universidad Politécnica de Valencia.

(2) Caturla, J.L. (1978). Compendio de los Sistemas Geodésicos en España. Publicación Técnica $n^{\circ}$ 1. Madrid: Instituto Geográfico Nacional.

(3) Dalda, A., Gonzalez, J. (2001). Parámetros de transformación entre sistemas de referencia. En Jornadas Técnicas sobre Topografía, Cartografía, Fotogrametría, Geodesia y Teledetección. Escuela de Arquitectura Técnica-Universidad de Alcalá.

(4) Consejo Superior Geográfico. (2007). Análisis de la problemática del cambio de datum geodésico a ETRS89. Madrid: Ministerio de Fomento.

(5) Consejo Superior Geográfico. (2007). Términos y definiciones de la ISO 19111. Madrid: Ministerio de Fomento.

(6) Cano, M., Talaya, J., Termens, A., Quirós, R., Revuelta, L., Sobrino, J. (2006). Ajuste de la Red Geodésica de Orden Inferior ROI de Cataluña en ETRS89. En $5^{a}$ Asamblea Hispano-Portuguesa de Geodesia y Geofísica. Sevilla, España.

(7) Bräker, F., Ebneter, F. (1997). The AlpTransit Project and the organisation of the survey. En FIG-Symposium. Denmark: Road Directorate.

(8) Fowler, S. (2006). Design and Preanalysis of Underground Control Networks for Tunnel Construction.

(9) Schödlbauer, D., Marti, U. (1997). The impact of the global positioning system on tunnel surveying. En FIG-Symposium Surveying of Large Bridge and Tunnel Projects. Kopenhagen.

(10) Martín, F.(1990). Geodesia y Cartografía Matemática. Madrid: Paraninfo.

(11) Snay, R.A. (1986). Network design strategies applicable to GPS surveys using three or four receivers. Journal of Geodesy, 60(1): 37-50.

(12) Unguendoli, M. (1990). A rational approach to the use of a large number of GPS receivers. Journal of Geodesy, 64(4): 303-312.

(13) Barbadillo, A., Quirós., M. (1996). Proyecto REGENTE. Una nueva Red Geodésica Nacional. Física de la Tierra, 8: 2333 .

(14) García, F., Olalla, D., Prieto, J. F., Velasco, J. (2009). Estudio del comportamiento de Multipath en receptores GPS en distintas condiciones (Proyecto Fin de Carrera). Madrid: E.T.S. de Ingenieros en Topografía, Geodesia y Cartografía.

(15) Nieto, J.J., García, F., Arranz, O., Velasco, J. (2000). Obervación de una red de lado medio por técnicas GPS con posterior cálculo y comparación de resultados (Proyecto Fin de Carrera). Madrid: E.T.S. de Ingenieros en Topografía, Geodesia y Cartografía.

(16) Chrzanowski, A. (1981). Optimization of the Breakthrough Accuracy in Tunneling Surveys. The Canadian Surveyor, 35: 5-16.

(17) Bjerhammar, A. (1973). Theory of errors and generalized matrix inverses. Amsterdam: Elsevier Scientific Publishing.

(18) Velasco, J., Prieto, J.F., Lopez, S. (2008). Establecimiento de la Red GNSS de InlanGeo en la Comunidad de Madrid: Aplicaciones en tiempo real. En VI Jornadas Internacionales de aplicaciones Geomáticas en Ingeniería. Madrid, España. 